\title{
The context principle in Frege's Grundgesetze
}

\author{
Øystein Linnebo
}

Draft of May 29, 2017

\section{Introduction}

Frege's context principle tells us 'never to ask for the meaning of a word in isolation, but only in the context of a sentence' (Frege, 1953, p. x). This principle is without doubt one of the cornerstones of Frege's Grundlagen. It is announced in the introduction as one of three 'fundamental principles' that Frege has kept in mind throughout this enquiry. The principle also plays an essential role in the ensuing discussion, especially that of how numbers and other abstract objects 'are given to us' (Frege, 1953, § 62).

By contrast, the context principle figures less prominently in Frege's magnum opus, the Grundgesetze. Indeed, there are reasons to doubt that any role remains for the context principle in the Grundgesetze - or so it appears. Firstly, Frege here subsumes the category of sentences under that of proper names. A sentence is now defined as a proper name that refers to a truth-value. As a result, it appears no longer to make sense to say that sentential contexts are privileged when it comes to explaining the meaning of a word. Secondly, the context principle appears to clash with the compositional semantics developed in the Grundgesetze and other works from the same period. While the context principle seeks to explain the meanings of subsentential expressions in terms of the meaning of entire sentences, the compositional semantics of Frege's mature period involves the opposite direction of explanation, where the meaning of any complex expression - including a sentence - is explained in terms of the meanings of its components. Finally, the Grundgesetze contains an extensive and vehement criticism of contextual definitions. It is unclear how the context principle can escape this attack.

The purpose of this article is to examine what role, if any, the context principle plays in 
the Grundgesetze. After an initial examination of the principle in the Grundlagen, I argue that - appearances to the contrary - the context principle retains an important role in the Grundgesetze. I also argue that reflection on this role sheds important new light on the context principle. More specifically, I argue that the context principle must be disentangled from the problematic idea of 'recarving of content'; that the principle is fully compatible with Frege's compositional semantics; and that it suggests a more 'lightweight' conception of mathematical objects than is typically associated with Frege.

Although it cannot be denied that Frege's discussion of the context principle is underdeveloped and partially flawed, I believe it merits our highest admiration. On my analysis, Frege articulates the important philosophical problem of our linguistic and cognitive 'access' to abstract objects such as numbers and develops a promising new answer based on the context principle. This contrasts favorably with other thinkers (such as Dedekind) who indulge in loose talk about 'the creation' of mathematical objects, and with yet others (such as the early Russell and at times Gödel) who invoke a largely unexplained kind of apprehension of an abstract but mind-independent reality. ${ }^{1}$ Frege's use of the context principle is a heroic, if only partially successful, attempt to face up to an extremely hard philosophical question, which so many other thinkers have largely evaded.

Some remarks about terminology are in order before we begin. In the Grundlagen, Frege never says much about the notion of meaning with which he is concerned; rather, he treats the notion in an informal way. In later works, however, Frege obviously has a lot to say about meaning. Beginning in the 1890s, he distinguishes sharply between sense and reference, and develops his influential theory of these notions and their interrelation. In what follows, I first rely on a single informal notion of meaning, much as in the Grundlagen. Later, it becomes necessary to consider more precise technical notions, in particular Frege's notions of sense and reference. To distinguish these different uses, I reserve the words 'sense' and 'reference' for Frege's two technical notions of Sinn and Bedeutung. The word 'meaning' will be used in part for the informal notion of the Grundlagen and in part as a placeholder for a more

\footnotetext{
${ }^{1}$ It is an interesting question, which I cannot discuss here, whether Frege's own late works, such as (Frege, 1956), also belong in this group. I have in mind Frege's robust realism about thoughts and his striking claims about our ability to grasp thoughts using "our power of thinking".
} 
precise notion yet to be spelled out.

\section{How are the numbers 'given to us'?}

Frege's context principle has received a bewildering range of interpretations. ${ }^{2}$ I believe the best way to understand the principle is by examining its contribution to the work where it figures most prominently, namely the Grundlagen. Since the main goal of this work is to defend a logicist account of mathematics, I shall be particularly concerned with the contribution that the context principle is supposed to make to Frege's logicism.

At Grundlagen $\S 62$ Frege raises a question that has dominated much of recent philosophy of mathematics:

How, then, are the numbers to be given to us, if we cannot have any ideas or intuitions of them? (Frege, 1953)

The problem is, of course, that numbers, unlike tables and chairs, cannot be perceived; nor can they be observed with the help of modern technology, as electrons and DNA molecules can. How can we then refer to numbers and other kinds of abstract objects, let alone gain knowledge of them? The question is perfectly reasonable, since the fact that a singular term or representation succeeds in referring to another object external to itself can hardly be primitive but must have some explanation.

Frege's next sentence proposes an answer to the above question:

Since it is only in the context of a sentence that words have any meaning, our problem becomes this: To explain [erklären] the sense of a sentence in which a number word occurs. ${ }^{3}$

Frege here proposes that his context principle has an essential role to play in the explanation of the meaning of individual words. In particular, since the meaning of a singular term is

\footnotetext{
${ }^{2}$ See (Pelletier, ) for a wide range of examples, as well as (Ebert, 2015) for an overview and critique of Dummett's view of the principle.

${ }^{3}$ I have changed the translation of 'Satz' from 'proposition' to 'sentence', which is reasonable given that Frege talks about words occurring in a Satz.

(Dummett, 1978, p. 38) characterizes this as 'probably the most important philosophical statement Frege ever made'. Later he uses even stronger words, describing it as 'arguably the most pregnant philosophical paragraph ever written' (Dummett, 1991a, p. 111). Although these are bold claims, it is not easy to think of better examples.
} 
closely related to - if not identical with - its reference, the context principle is relevant to the question of how the numbers are 'given to us'. The idea is to transform the hard problem of explaining how an arithmetical term succeeds in referring into the easier problem of explaining the meanings of certain complete sentences involving this term.

But is the latter problem really any easier than the former? When we explain the meaning of a sentence, must we not also explain the reference of every singular term involved in the sentence? If so, Frege will only have transformed one hard problem into another that is at least as hard. Frege is fully aware of this worry and responds that, when we explain the meaning of each of the relevant sentences, 'we must reproduce the content of this sentence in other terms, avoiding the use of' the singular terms whose reference we are trying to explain $(\S 62)$. Our task is thus to 'reproduce' the meaning of each sentence containing the problematic singular terms in a way that avoids use of these terms. Frege provides a useful example of what he has in mind. He suggests that the identity statement 'the direction of line $a$ is identical with the direction of line $b$ '-which contains problematic singular terms purporting to refer to directions - has a content that can be 'reproduced' by means of the sentence 'line $a$ is parallel to line $b$ - which avoids the problematic direction terms and relies only on the simpler phenomenon of reference to lines.

How might such a 'reproduction of meaning' help us explain reference to a direction? One would have thought that any account of reference to some object would have to mention this object! But the proposed 'reproduction of meaning' is chosen precisely because it avoids any mention of directions. Of all the questions surrounding the context principle, this one may well be the hardest - as will become abundantly clear in what follows.

To sum up, Frege's response to the question of how the numbers are 'given to us' involves two key moves: first, the context principle, which transforms a question about the reference of certain problematic singular terms into a question about the meanings of complete sentences containing these term; then, an answer to the latter question based on the idea of 'reproducing' the meaning of each sentence containing the problematic singular terms in a way that avoids any use of these terms. The next two sections consider each move in turn. 


\section{The context principle in the Grundlagen}

The context principle receives four different statements in the Grundlagen, of which the first and third (as ordered by their occurrence in the book) have already been quoted.

CP1. never ask for the meaning of a word in isolation, but only in the context of a sentence (p. x)

CP2. Only in a sentence have the words really a meaning. [...] It is enough if the sentence taken as a whole has sense; in this way its parts too obtain their content. $(\S 60)$

CP3. Since it is only in the context of a sentence that words have any meaning, our problem becomes this: to define [erklären] the sense of a sentence in which a number word occurs. $(\S 62)$

CP4. We next laid down the fundamental principle that we must never try to explain [erklären] the meaning of a word in isolation, but only as it is used in the context of a sentence. $(\S 106)$

How should these passages be interpreted?

Many commentators take the context principle to consist, at least in part, in the claim that individual words never have any meaning in isolation and that only complete sentences are capable of possessing an independent meaning. ${ }^{4}$ Thus, they interpret Frege as endorsing the following principle.

\section{A necessary condition for possession of meaning}

If a subsentential expression $e$ is meaningful, then $e$ occurs in a meaningful sentence.

This interpretation is supported by the first half of each of CP2 and CP3. Further evidence is provided by $\S 60$, where Frege writes that 'The self-subsistence which I am claiming for

\footnotetext{
${ }^{4}$ Quine is an influential exponent of this interpretation; see e.g. (Quine, 1953, p. 39); see also (Resnik, 1967), (Resnik, 1976) (who on p. 42 makes the even stronger claim that 'sentences but not words have meaning'), and (Janssen, 2001). Yet further examples are discussed in (Pelletier, ).
} 
number is not taken to mean that a number word signifies something when removed from the context of a sentence.'

However, this necessary condition seems unreasonably strong. ${ }^{5}$ To see this, let a unit of significance be the smallest linguistic string that has meaning in isolation. Then the condition says that the only units of significance are complete sentences, never individual words. ${ }^{6}$ Individual words have no meaning whatsoever when they occur outside of sentential contexts. But this seems to contradict one of the most important and least controversial principles of linguistics, namely that words have independent lexical meanings that play an important role in determining the meanings of sentences in which they occur. ${ }^{7}$

In fact, throughout most of his career, Frege was firmly committed to the idea that words have independent meanings. This becomes abundantly clear in Frege's mature work, where (as we shall see in Section 5) he endorses a principle of compositionality based on exactly this idea. But already in his early work, we find passages sympathetic to the idea. For example, in the unpublished article 'Boole's logical calculus and the Concept-scrip' from 1880-81, Frege writes that in his logically perfect language (Begriffsschrift) the 'designations' of properties and relations

never occur on their own, but always in combinations which express contents of possible judgment. I could compare this with the behavior of the atom: we suppose an atom never to be found on its own, but only combined with others, moving out of one combination only in order to enter immediately into another. (Frege, 1979, p. 17) ${ }^{8}$

This passage suggests a weaker and far more plausible view than that sentences are the only units of significance. Words have independent 'contents' or meanings, much like atoms have independent properties. However, these meanings cannot 'occur on their own' but only in the

\footnotetext{
${ }^{5}$ Here I follow a long tradition that includes (Dummett, 1956), (Dummett, 1981a, ch. 1), and (Dummett, 1981b, ch. 19).

${ }^{6}$ Unless the individual word also functions as a sentence, such as the Latin 'cogito' or the Spanish 'pienso' (cf. (Dummett, 1956, p. 492)). Henceforth, this qualification will be tacitly assumed.

${ }^{7}$ Of course, ambiguities need to be resolved, and this will often require information from the broader context. Henceforth, we shall assume that all ambiguities have already been eliminated (as Frege too assumed concerning his Begriffsschrift).

${ }^{8}$ Frege can be excused for not knowing that Helium and other inert gases falsify his chemical 'supposition', as Helium was isolated only in 1895. The exact empirical facts are anyway irrelevant to Frege's logical point.
} 
context of a complete judgment, much like atoms (typically) cannot occur on their own but only bound together in a molecule.

Is it possible to reconcile this apparent acceptance of subsentential units of significance with the passages where Frege seems to endorse the necessary condition? One attractive possibility emerges when we raise an important question that has so far been ignored. Are the mentioned passaged concerned with expression types or tokens? Our complaint that the condition is unreasonably strong tacitly assumes that the expressions in question are tokens. An expression token - such as a word scribbled on a piece of paper-can (quite literally) be moved in and out of sentential contexts, while throughout this process contributing its fixed meaning to the resulting range of sentential meanings. Now, suppose instead that the quoted passages are concerned with expression types. Thus understood, the necessary condition states that no subsentential expression type can be meaningful unless it occurs in a meaningful sentence type. But since every expression type occurs in an infinite range of sentence types, this is no longer such a demanding requirement. Indeed, provided that a subsentential expression token is so much as capable of occurring in a meaningful sentence token, then presumably the corresponding expression type in fact occurs in the corresponding sentence type, which is of course also meaningful. Thus, the proposed interpretation would reconcile the two apparently conflicting pressures with which we began. This provides at least some support for the interpretation. ${ }^{9}$

Regardless of one's view of this interpretation, however, it is clear that the necessary condition cannot be all there is to the context principle. While some of the quoted passages suggest the necessary condition, others suggest other principles. In particular, the second half of each of CP2 and CP3 suggest the following sufficient condition:

\section{A sufficient condition for possession of meaning}

If a subsentential expression $e$ occurs in a meaningful sentence, or in some appropriate family of meaningful sentences, then $e$ is meaningful.

In fact, this sufficient condition makes far more sense in the dialectical context in which the context principle occurs. As we saw in the previous section, the principle is invoked

\footnotetext{
${ }^{9}$ Further support becomes available in the Grundgesetze, as explained in footnote 21.
} 
in order to transform a hard problem concerning reference to numbers and other abstract objects into an easier problem concerning the meanings of certain complete sentences. This explanatory strategy requires that the meaning or reference of a singular term can be explained by observations concerning the meaning of certain complete sentences. This move-from sentential meaning to subsentential meaning - can be supported by the sufficient condition. By contrast, necessary condition - even on its more plausible type-based interpretation - is plainly irrelevant to the mentioned explanatory move and the broader dialectical context in which the passages occur. ${ }^{10}$ I conclude that there is at least as strong a case for taking Frege to endorse the sufficient condition as the necessary condition.

In fact, Frege might have taken both conditions to follow from a single underlying idea. One reason to think so concerns a remarkable feature of CP2 and CP3, which we have not yet discussed. Both passages move unannounced from what appears to be statements of the necessary condition to a conclusion that requires the sufficient condition. Consider, for example, CP3. Why should it follow from the necessary condition that "our problem become [... to define [erklären] the sense of a sentence in which a number word occurs"? If anything, this inference requires the sufficient condition, not the necessary one! So unless Frege is relying on some broader idea of contextuality, the arguments in CP2 and CP3 would be obvious non sequiturs.

Another reason concerns the two remaining passages, namely $\mathrm{CP} 1$ and $\mathrm{CP} 4$, which in fact express a broader idea of contextuality. ${ }^{11}$

\section{Sentence-level explanation of meaning}

The meaning of a subsentential expression $e$ can only be explained in terms of its contribution to the meaning of sentences in which $e$ occurs.

I use the word 'explain' to echo Frege's 'erklären'. While it is not entirely clear what kind of explanation Frege had in mind, the basic idea is tolerably clear. A meaningful expression makes some systematic contribution to the meanings of sentences in which the expression

\footnotetext{
${ }^{10}$ The same goes for the statements of the context principle that are found in the Grundgesetze, as we shall see.

${ }^{11}$ Cf. (Burge, 2005, p. 15), who distinguishes three interpretations of the context principle that are close to the ones articulated here.
} 
occurs. But this contribution cannot be fully characterized on its own but only in terms of its effect on the resulting sentential meanings. Thus, it is useful to think of the meaning of the subsentential expression as a capacity to affect or contribute to the meaning of sentences in which the expression occurs. This capacity cannot be fully explained in isolation but only in terms of its effect on sentential meaning. As Frege suggests in $\S 59$, the word 'only' provides a good example. The word clearly contributes in a systematic way to the meanings of complete sentences. But it makes no sense to try to explain or characterize its meaning in isolation, without any mention of complete sentences.

I claim that the explanatory principle coheres well with — and probably even supports - the necessary and sufficient conditions for possession of meaning. Suppose, with the explanatory principle, that for a subsentential expression to be meaningful is for it to be capable of making a systematic contribution to sentential meaning. Thus, if a subsentential expression $e$ occurs in a meaningful sentence, or in some appropriate family of meaningful sentences, then $e$ has the mentioned capacity and thus is meaningful. This yields the sufficient condition. Next, if the expression $e$ is meaningful, then its capacity to contribution to sentential meaning must be capable of manifestation. If the expressions in question are types, not tokens, then this ensures that $e$ in fact figures in meaningful sentences. This yields the necessary condition.

In sum, while Frege's statements of the context principle are not entirely clear, I have argued that the primary idea is an explanatory principle to the effect that the meaning of a subsentential expression can be properly explained only in terms of its contribution to the meaning of complete sentences. The statements of the context principle also suggests a necessary and (especially) a sufficient condition for possession of meaning. But reasonable versions of these conditions are supported by the primary idea.

\section{The 'reproduction' of meaning}

Let us now take a closer look at Frege's idea of 'reproducing' the meaning of a sentence containing singular terms that purport to stand for numbers (or some other kind of abstract objects) in a way that avoids using such terms. By $\S 62$ of the Grundlagen, Frege has already argued that number words stand for 'self-subsistent objects'. 'And that is enough to give us', 
he says, 'a class of sentences' whose meanings must be explained, namely identity statements involving number words (Frege, 1953, §62). He continues:

In our present case, we have to explain the meaning ['Sinn'] of the sentence 'the number which belongs to the concept $F$ is the same as that which belongs to the concept $G^{\prime}$; that is to say, we must reproduce the content of this sentence in other terms, avoiding the use of the expression 'the number which belongs to the concept $F^{\prime}$.

In fact, the 'number words' that Frege has in mind have a very specific form. Earlier in the Grundlagen, he has argued that numbers are ascribed to concepts; for example, the statement 'there are eight planets' should be analyzed as an ascription of the number eight to the concept is a planet. The basic number words are therefore taken to be of the form 'the number of $F$ s', where $F$ is a concept term; I shall abbreviate these terms as ' $\# F$ '. Thus, Frege takes our initial task to be to explain the meanings of numerical identity statements of the form ' $\# F=\# G$ '.

What about the meanings of sentences other than identities? Do these too have to be explained? There is evidence that Frege thought so. For in $\S 65$, he comments on the need to 'define' all other assertions about the relevant objects, not just identities. Moreover, in the 'generalized context principle' of the Grundgesetze, to be discussed shortly, Frege attaches no special significance to identity statements but holds that, in order to ensure that a singular term refers, all sentences in which the term occurs must be assigned truth-conditions.

As we have seen, the desired explanation of meaning is one that 'reproduces' the content or meaning of a sentence in other, and less problematic, terms. Let us call this the elimination requirement. When we 'reproduce' the meaning of a basic numerical identity, for example, we must avoid any use of the number terms that flank the identity sign. Can the elimination requirement be met? In particular, can we explain the meaning of basic numerical identities without any use of number terms? Frege believes that we can. His argument is based on a principle that he attributes to Hume - and which has consequently become known as Hume's Principle ${ }^{12}$ - namely that the number of $F \mathrm{~s}$ is identical to the number of $G \mathrm{~s}$ if and only if

\footnotetext{
${ }^{12}$ Although the principle was put to systematic use only much later by mathematicians, such as Georg
} 
the $F$ s and the $G$ s can be one-to-one correlated. We can formalize this as follows:

$$
\# F=\# G \leftrightarrow F \approx G
$$

where $F \approx G$ is some second-order formalization of the claim that there is a relation that one-to-one correlates the $F \mathrm{~s}$ and the $G \mathrm{~s} .{ }^{13}$ Frege's proposal is that instances of the right-hand side of (HP) can be used to explain the meaning of corresponding instances of the left-hand side. And this explanation appears to satisfy the elimination requirement.

In fact, the explanation seems to generalize to other abstraction principles as well, that is, to principles of the form:

$$
\S \alpha=\S \beta \leftrightarrow \alpha \sim \beta,
$$

where $\alpha$ and $\beta$ are variables, $\S$ is an operator that takes such variables to singular terms, and $\sim$ is an equivalence relation on the kind of entities that $\alpha$ and $\beta$ range over. Frege's claim about 'reproduction of meaning' seems to generalize as well. An instance of the right-hand side can be seen as 'reproducing' the meaning of the matching instance of the left-hand side in a way that also appears to satisfy the elimination requirement.

Unfortunately, things are not as simple as they appear. It is true that there is no explicit use of number terms on the right-hand side of (HP). Even so, there is an implicit reliance on such terms. Let us say that an abstraction principle is impredicative if its right-hand side quantifies over the objects to which its left-hand side refers. For instance, (HP) is impredicative because the first-order quantifiers on its right-hand side have numbers in their range. Although the right-hand side of (HP) makes no explicit use of number terms, it uses quantifiers whose permissible instances include such terms. Is this implicit reliance on number terms compatible with the elimination requirement? In other work, I defend a

${ }^{13}$ Hume's Principle is now known to have an amazing mathematical property. Call the second-order theory with (HP) as its sole non-logical axiom Frege Arithmetic. Then a technical result known as Frege's Theorem says that Frege Arithmetic, along with some very natural definitions, allows us to derive all of second-order Peano Arithmetic. This result is hinted at in (Parsons, 1965) and explicitly stated and discussed in (Wright, 1983). For a nice proof, see (Boolos, 1990).
} 
negative answer. ${ }^{14}$ For present purposes, we may simply sidestep the complication posed by impredicative abstraction principles by testing the ideas associated with the context principle on predicative principles, where the complication does not arise.

Our next question is how Frege understands the desired 'reproduction' of meaning. He makes some interesting remarks in $\S 64$, in the context of discussing the example of directions. Since two directions are identical just in case the lines whose directions they are, are parallel, we get the following abstraction principle for directions:

$$
d(a)=d(b) \leftrightarrow a \| b
$$

where $a$ and $b$ range over directions or other directed items. Frege writes: ${ }^{15}$

Thus we replace the symbol $\|$ by the more generic symbol $=$, through removing what is specific in the content of the former and dividing it between $a$ and $b$. We carve up the content in a way different from the original way, and this yields us a new concept. (Frege, 1953, §64)

The suggestion is that each instance of the right-hand side of (Dir) 'recarves' the content of the corresponding instance of the left-hand side. That is, the two sides share a common content or meaning, which they 'carve up' in different ways. ${ }^{16}$ However, there are exegetical questions about the depth and strength of Frege's commitment to the idea. As is well known, Frege abandons the approach based on recarving in the Grundlagen $\S 68$ in favor of an explicit definition of numbers in terms of extensions. The context principle is nevertheless reiterated as a 'fundamental principle' at the end of the book (§106). This suggests that Frege already in the Grundlagen is more strongly committed to the context principle than to the idea of recarving. ${ }^{17}$ As we shall see shortly, this differential commitment becomes indisputable in the

\footnotetext{
${ }^{14}$ See (Linnebo, , ch. 6). Our present question - whether impredicative abstraction is compatible with the elimination requirement - must not be confused with the more general question about the permissibility of impredicative abstraction principles. On the latter question, see e.g. (Dummett, 1991a) and (Dummett, 1998), who finds such principles problematic, and (Wright, 1998a) and (Wright, 1998b), who does not.

${ }^{15}$ See also the passage from $\S 65$ quoted on the next page.

${ }^{16}$ This 'recarving thesis' is also endorsed by Bob Hale and Crispin Wright in (Wright, 1983) and in many of the articles collected in (Hale and Wright, 2001), esp. essay 4 (originally (Hale, 1997)) but also essay 5, pp. 149-150; essay 8, pp. 192-197; and essay 12, pp. 277-278. However, in more recent work by Hale and Wright, the recarving thesis plays a less central role; see e.g. (Hale and Wright, 2000) and (Hale, 2007).

${ }^{17}$ Thanks to Philip Ebert for this suggestion.
} 


\section{Grundgesetze.}

Let us take stock of our discussion so far. We have obtained a good understanding of the architecture of Frege's strategy in the Grundlagen for explaining how numbers are 'given to us'. The strategy relies on two main ingredients - the context principle and the idea of 'reproducing' meaning in other, and less problematic, terms-which are supposed to work together in tandem. While the overall strategy is thus fairly clear, the details of its execution are less so. Our next task is to examine whether the context principle survives into the Grundgesetze and, if so, whether this can help us attain a better understanding of how Frege's strategy was supposed to be executed.

\section{The context principle in the Grundgesetze}

Many commentators claim that Frege gave up the context principle at some point between the Grundlagen and the Grundgesetze. ${ }^{18}$ Three arguments for this claim were identified in the introduction. The purpose of this section is to show that all three arguments fail, and that, on the contrary, the context principle retains an essential role in the argument of the Grundgesetze.

The first argument concerns Frege's subsumption of the category of sentences under that of proper names. In the Grundlagen, Frege still adheres to the more traditional — and plausibleview that sentences and proper names are two separate logical categories. The context principle relies on this fact, as it asserts an explanatory priority of the former category over the latter. By contrast, the Grundgesetze regards a sentence as merely a complex proper name that refers to a truth-value. This obliterates the earlier distinction between sentences and proper names on which the earlier version of the context principle was based. It is thus

\footnotetext{
${ }^{18}$ See (Resnik, 1967) and (Resnik, 1976) for expressions of this view. (Janssen, 2001, p. 133) makes the slightly weaker claim that after the Grundlagen, 'the context principle was not repeated in [Frege's] published writings'. See (Künne, 2010, p. 595) for a similar claim, as well as (Pelletier, ) for further examples. Some of Dummett's writings may suggest that he agrees; see e.g. (Dummett, 1981a, pp. 7 and 495). However, the relevant passages are more plausibly read as observing that, as of the 1890s, Frege can no longer regard sentential contexts as privileged because he now thinks that sentences are a special kind of proper names. Despite this, Dummett claims that Frege 'retains the context principle [...] as far as can be done without distinguishing between sentences and other complex proper names' (Dummett, 1981b, p. 409). See also (Dummett, 1981b, ch. 19), (Dummett, 1991a, ch. 17), (Dummett, 1991b, pp. 229-233), and (Dummett, 1995).
} 
unclear whether the principle can even be formulated in the context of the Grundgesetze.

However, a principle very much like the original context principle remains in the Grundgesetze. For example, in $\S 97$ of volume II we read: 'One can ask after reference only where signs are components of propositions expressing thoughts.' As this passage and a number of others illustrate, the subsumption of sentences under proper names in no way undermines Frege's notion of a thought or his emphasis on judgment. ${ }^{19}$ The most important evidence is found in Grundgesetze I $\S \S 29-31$, where Frege makes heavy use of a contextual account of reference, sometimes known as the 'generalized context principle'. This account lays down the following criterion for a proper name to refer: ${ }^{20}$

A proper name has reference if, whenever it fills the argument places of a referential name of a first-level function with one argument, the resulting proper name has a reference, and if the name of a first-level function with one argument which results from the relevant proper name's filling the $\xi$-argument-places of a referential name of a first-level function with two argument places, always has a reference, and if the same also holds for the $\zeta$-argument-places. (Frege, 2013, I, §29)

Although the passage is highly compressed, the basic picture is tolerably clear. With the subsumption of the category of sentences under that of proper names, the context principle becomes a principle to the effect that a proper name is referential provided that all larger contexts in which the name occurs are themselves referential-including contexts that belong to the category of proper names. So the central idea of the context principle remains unchanged, namely that a proper name refers provided that it contributes appropriately to the meanings of all larger contexts in which it occurs. ${ }^{21}$

The quoted criterion for a proper name to refer appeals to a notion of a function name's referring, for which Frege provides the following criterion:

\footnotetext{
${ }^{19}$ Rather, what has happened is that the distinctive characteristic of sentences - namely their role in assertion - has been separated out and located in Frege's assertion stroke, where $\vdash \Delta$ indicates the judgment that $\Delta$ is the True. See (Rumfitt, 2011).

${ }^{20}$ See (Heck, Jr., 1997), (Heck, Jr., 2012, ch.s 3 and 5), and (Linnebo, 2004) for further discussion.

${ }^{21}$ In Section 3, I asked whether the expressions with which the context principle is concerned should be understood as types or tokens. I provided some evidence for the former understanding. Further evidence is provided by the generalized context principle, which becomes unstable unless this understanding is adopted. Suppose more token names of first-level functions become available as time goes by. This may cause a proper name that was initially deemed referential to no longer be so.
} 
A name of a first-level function with one argument has a reference [...] if the proper name which results from this function-name when the argument places are filled by a proper name always has a reference provided the inserted name refers to something. (ibid.)

Frege also states criteria for names of first-level functions of two arguments to refer and for names of second- and third-level functions. These criteria are obvious modifications of the two just quoted and need not be reproduced here. ${ }^{22}$

Clearly, to use these criteria of referentiality, we need a way to verify that a complex name refers that does not depend on a prior verification of the reference of its parts. How might this be possible? Frege's attempted 'proof of referentiality' in $\S \S 29-31$ reveals what he had in mind. The idea of the proof is to reduce a complex name to another name that is guaranteed to corefer, if it refers at all, but whose referentiality can be established more easily. A nice example is provided by Frege's horizontal function $-\xi$, which is defined to map the True to the True and everything else to the False. A moment's reflection shows that this name is guaranteed to corefer with the complex name ' $\xi=(\xi=\xi)$ '. Thus, if the name of the identity function refers, so does that of the horizontal function. Indeed, Frege shows that the proof of referentiality comes down to verifying the referentiality of all identities involving value-range terms from the language of the Begriffsschrift. And this is meant to follow from another reduction of the prescribed sort, this time involving a metalinguistic version of Basic Law V:

I use the words

"the function $\varphi(\xi)$ has the same value-range as the function $\Psi(\xi)$ "

throughout as co-referential with the words

"the functions $\varphi(\xi)$ and $\Psi(\xi)$ always has the same value for the same argument."

\footnotetext{
${ }^{22}$ Note the circular dependence of the two criteria: each makes ineliminable reference to the other. For discussion, see (Linnebo, 2004), where this problem is referred to as 'the old circularity', as it also affects the context principle from the Grundlagen. It is relevant, in this connection, that Frege notes that his criteria 'are not to be regarded as explanations of the expressions 'to have a reference' or 'to refer to something', since their application always presupposes that one has already recognised some names as referential; but they can serve to widen the circle of such names gradually' (Frege, 2013, I, §30). I believe this stepwise use of Frege's criteria corresponds to a sequence of expansions of one's linguistic resources of the sort to be discussed in Section 6.4.
} 
(Frege, 2013, I, §3)

The importance of this passage is highlighted by the fact that it opens the section of the Grundgesetze where value-ranges are introduced. But Frege's proof of referentiality must be flawed. For by Russell's paradox, Frege's stipulations cannot ensure that every name of the Begriffsschrift is assigned a unique reference.

Not all is lost, however. There is no problem provided that we restrict ourselves to the predicative version of Basic Law V. ${ }^{23}$

As we have seen, the context principle was part of Frege's attempt in the Grundlagen to explain our cognitive 'access' to numbers and other abstract objects. The same philosophical concern arises in the Grundgesetze, as the following passage makes clear. ${ }^{24}$

If there are logical objects at all — and the objects of arithmetic are such — then there must also be a means to grasp them, to recognise them. (Frege, 2013, II, $\S 147)$

Frege's attempted explanation too has the same structure as in the Grundlagen. Speaking of the 'transformation' afforded by the metalinguistic version of Basic Law V that we quoted above, the above passage continues:

The basic logical law which permits the transformation of the generality of an equality into an equality serves for this purpose. Without such a means, a scientific justification of arithmetic would be impossible. For us it serves the purposes that other mathematicians intend to achieve by the creation of new numbers.

Let us assemble all the pieces. In both of his main works, Frege recognizes the need to explain how we grasp abstract objects such as numbers. And in both works, he attempts to meet this challenge by providing a two-step explanation of the sort we identified in the

\footnotetext{
${ }^{23}$ Other fragments can be salvaged as well. (?) show how a version of the groundedness idea guiding (Kripke, 1975) can successfully be brought to bear on the impredicative version of Basic Law V. Unfortunately, this method works only when the background second-order logic is predicative. (Dummett, 1991a, ch. 17) is thus half right when he characterizes impredicativity as the serpent in Frege's paradise. Although each of the two kinds of impredicativity can be handled in a satisfactory way, it is impossible to do so for both kinds simultaneously.

${ }^{24} \mathrm{~A}$ similar passage is found at the very end of the postscript that Frege added to the book, where this is characterized as the 'Urproblem' of arithmetic.
} 
Grundlagen. First, the context principle is meant to transform the problem of explaining the reference of a class of problematic proper names to that of explaining the meanings of more complex expressions in which these names occur. Then, this latter problem is meant to be solved by showing how the meanings of these more complex expressions can be 'reproduced' without any use of the mentioned class of names. As one would expect, given the developments in Frege's view of semantics, the exact nature of each step differs between the two works. In the Grundlagen, the first step amounts to the task of explaining the meanings of sentences in which the problematic proper names occur, whereas in the Grundgesetze, we face the task of explaining the reference of more complex names containing the problematic proper names as parts. Likewise, the 'reproduction' of meaning involved in the second step is understood differently in the two works. In the Grundlagen, the 'reproduction' is one of 'recarving', that is, of expressing the very same meaning in a different way; whereas in the Grundgesetze, it is simply a matter of reducing a complex expression to simpler yet co-referring one, as illustrated by the passage quoted from Grundgesetze I, $§ 3$. The following table provides a summary (where the arrow $\rightsquigarrow$ indicates a reduction of one problem to another).

\begin{tabular}{|l|l|l|}
\hline \multicolumn{2}{|c|}{ Step 1: Transformation of the problem } \\
\hline $\begin{array}{l}\text { Grundlagen } \\
\text { Grundgesetze }\end{array}$ & reference of proper names $\rightsquigarrow$ & meaning of sentences \\
& $\rightsquigarrow$ reference of more complex names \\
Step 2: 'Reproduction' of meaning that avoids the problematic names \\
\hline $\begin{array}{l}\text { Grundlagen } \\
\text { Grundgesetze }\end{array}$ & 'recarving' of meaning \\
\hline
\end{tabular}

I turn now to the second of the three arguments aimed to show that the context principle is incompatible with Frege's view in the Grundgesetze. This argument is concerned with the compatibility of the context principle with the principle of compositionality, which figures prominently in Frege's middle and later works. The semantic theory developed in this period has at its core a two-place relation of reference that holds between a linguistic expression and 
the worldly item that serves as its Bedeutung or semantic value. ${ }^{25}$ One of Frege's great innovations is to provide a systematic account of how the semantic value of a complex expression is related to the semantic values of its subexpressions. In particular, he formulates the principle of compositionality, which says that the semantic value of a complex expression is determined as a function from the semantic values of its immediate parts. According to Frege, the syntactic operation of predication corresponds to the semantic operation of function application. So the semantic value of the simple predication 'John runs' is obtained by applying to the semantic value of 'John' the semantic value of 'runs'. If we write $\llbracket \rrbracket$ for the semantic value of an expression $e$, then the analysis of the simple predication can be formalized as follows:

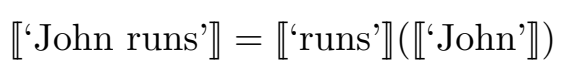

Frege endorses an even stronger compositionality principle for senses. When one expression is a syntactic part of another, then the sense of the former is claimed to be a part of the sense of the latter. ${ }^{26}$

We now face a puzzle. How can the context principle be reconciled with the principle of compositionality? After all, the two principles call for opposite forms of explanation. The principle of compositionality has a bottom-up character: the semantic properties of complex expressions are to be explained in terms of the semantic properties of their parts. By contrast, the context principle has a top-down character: a key semantic property of proper namesnamely their reference - is to be explained in terms of the semantic properties of more complex expressions in which the relevant names occur. ${ }^{27}$

In fact, the puzzle has a solution. There would indeed be a conflict if, as some commentators claim, the context principle denied that individual words can be units of significance. For when words compose to form a complex expression, then compositionality, as ordinarily

\footnotetext{
${ }^{25}$ Strictly speaking, we need a family of reference relations, one for each of Frege's fundamental ontological categories. For example, the relation of reference that obtains between a proper name and the object to which it refers is not the same as that which obtains between a monadic first-level predicate and the first-level function of one argument. For ease of exposition, I henceforth suppress this complication.

${ }^{26}$ See for instance (Frege, 2013, I, §32) and (Frege, 1963).

${ }^{27}$ The alleged conflict is nicely summarized by (Resnik, 1976, p. 47), who writes that with compositionality, ' $[\mathrm{t}$ ] he meaning of the sentence rather than bestowing meaning upon the words becomes a function of the meanings of the words.' See also (Pelletier, ) for a wealth of examples of thinkers who believe there is at least a prima facie tension between the two principles.
} 
understood, tells us that the meaning of the latter can be determined on the basis of meanings antecedently possessed by the former. But the mentioned interpretation of the context principle is not well supported. As argued above, the purpose of the principle, as it figures in the Grundlagen, is not to deny that words can be units of significance but rather to insist that their meaning or significance can be properly explained only in terms of their contribution to the meanings of complete sentences. Moreover, the mentioned interpretation lacks any support from the Grundgesetze, where the context principle figures solely as a sufficient condition for possession of meaning and as an explanatory principle.

Might either the sufficient condition or the explanatory principle conflict with the principle of compositionality? There is certainly nothing to fear from the sufficient condition, which in fact goes hand in hand with compositionality. For unless an expression $e$ has a semantic value, one could not in a compositional way obtain a semantic value for any complex expression in which $e$ occurs. But let us take a closer look at the explanatory principle, which, we recall, states that the meaning of certain expressions can only be explained in terms of their contribution to the meanings of more complex expressions. There is no direct conflict between this principle and the principle of compositionality, which states that the meanings of complex expressions are determined by the meanings of their simpler constituents. While the former principle concerns the explanation of meaning, the latter concerns the determination of meaning. ${ }^{28}$ The only worry is that some indirect conflict might emerge once the top-down explanation promised by the context principle is properly spelled out. Although this is theoretically possible, no concrete reason for suspicion has been provided - or emerges when we shortly consider some attempts to spell out the details.

The final argument that Frege cannot have retained the context principle in the Grundgesetze is concerned with his scathing criticism of creative definitions, especially in Grundgesetze II, $\S \S 138-146$. Since the targeted definitions include what have become known as contextual definitions, it is natural to think that Frege's criticism must have made him reject the context principle. Some relevant text occurs at the end of Frege's discussion of creative definitions, which concludes that it 'has thus become plausible that creating proper is not available to

\footnotetext{
${ }^{28}$ Compare (Dummett, 1981a)'s response to the alleged conflict, which is based on a distinction between 'the order of explanation' of meaning and 'the order of recognition' (pp. 4ff).
} 
the mathematician' (§146). In the very next sentence, Frege considers an objection:

Against this, it could be pointed out that in the first volume $(\S 3, \S 9, \S 10)$ we ourselves created new objects, namely value-ranges. What did we in fact do there? Or to begin with: what did we not do? We did not list properties and then say: we create a thing that has these properties. Rather, we said: if one function $[\ldots]$ and a second function are so constituted that both always have the same value for the same argument, then one may say instead: the value-range of the first function is the same as the value-range of the second. That [...] we can convert the generality of an equality into an equality (identity), must be regarded as a logical basic law. This conversion is not to be taken as a definition; neither the words "the same", nor the equality-sign, nor the expression "value-range", nor a combination of [value-range signs], nor both at the same time, are thereby explained. (Frege, 2013, §146).

I believe the 'conversion' that Frege has in mind is the metalinguistic version of Basic Law V. (Had it been the ordinary version of the law, there would have been no possibility of confusing it with a definition.) This prompts the question of why the conversion isn't an illegitimate creative definition. The very fact that Frege raises this question shows that he is aware of the apparent tension between his criticism of creative definitions on the one hand, and the context principle and its use of the mentioned conversion on the other. His answer is that the conversion isn't a definition at all, and that the apparent tension therefore isn't genuine. For our present purposes, it immaterial whether the answer is a good one. What matters is that Frege identifies the question and proposes an answer. This suffices to undermine the thought that Frege's criticism of creative definitions must have forced him to reject the context principle. This completes my response to the final argument. ${ }^{29}$

To sum up, I have argued that both the context principle and the explanatory project in which the principle figures survive into the Grundgesetze, subject only to the changes required

\footnotetext{
${ }^{29}$ Although not required by my response, it is hard to resist asking what the status of Frege's conversion is, if not a definition. We are told that it 'must be regarded as a logical basic law'. But this is not very helpful, as this can at best be said about the ordinary version of Basic Law V, not about the metalinguistic version. We shall return to this question shortly.
} 
by other developments in Frege's view, especially concerning semantics.

\section{Developing Frege's explanatory strategy}

So far I have focussed on the architecture of the explanatory strategy associated with the context principle, as this principle figures in Frege's two main works. It is time to delve into the nuts and bolts, both in order to understand how Frege wanted to arrange them, but also to investigate whether the strategy can in fact be implemented. I shall consider three attempts to develop the explanatory strategy, two inspired by the Grundlagen, and a third, by the Grundgesetze. Since the third strategy is the most promising, this illustrates the importance of not limiting our investigation of the context principle to its familiar home in the Grundlagen.

\subsection{Our test case in more detail}

Recall our decision to begin by testing the ideas associated with the context principle on the technically simple family of predicative abstraction principles. It suffices to consider Frege's own example of directions. The generalization to other predicative abstraction principles is immediate. Although this material is part of the folklore, it will be useful to develop this test case properly. Some of the details are subtle and rarely given a careful presentation.

Let $\mathcal{L}_{0}$ be an interpreted language with vocabulary appropriate for discourse about (among other things) lines. Let $\mathcal{L}_{1}$ be the result of adding to $\mathcal{L}_{0}$ vocabulary appropriate for discourse about (among other things) directions. This includes an abstraction operator $\S$, which is associated with an equivalence relation $\sim$. Thus, in our canonical example of directions, $\S a$ is intended to stand for the direction of a line $a$, and $\sim$ is parallelism. The vocabulary that is added to $\mathcal{L}_{1}$ is not assumed to be interpreted; rather, the idea is to use the context principle to explain how the new expressions come to be meaningful. As we have seen, Frege's strategy is to 'reproduce' the meaning of each sentence of the extended language in a way that avoids using the vocabulary whose meaning we are trying to explain. It is plausible to interpret this as an attempt to 'reproduce' the meaning of each sentence of $\mathcal{L}_{1}$ by means of a sentence of $\mathcal{L}_{0}$. This corresponds to defining a translation $\tau$ from sentences of $\mathcal{L}_{1}$ to sentences $\mathcal{L}_{0}$. 
To define such a translation, it is useful to let $\mathcal{L}_{1}$ be a two-sorted language, with one sort for lines and another for directions. This means that problematic 'mixed' identities, involving one direction term and one line term, cannot even be formulated. We thus postpone the notorious Caesar-problem, which is independent of our present task of interpreting the context principle. The desired translation $\tau$ from $\mathcal{L}_{1}$ to $\mathcal{L}_{0}$ can now be defined. The basic idea is simple. ${ }^{30}$ Any formula of $\mathcal{L}_{1}$ that consists entirely of line vocabulary is translated by $\tau$ as itself. Any identity of the form $\S a=\S b$ is translated as $a \sim b$. What about atomic formulas involving direction predicates? Here we use the fact that all such predicates can be obtained from line predicates which do not discriminate between parallel lines; for example, the orthogonality predicate $\perp^{*}$ is obtained from the predicate $\perp$ that holds between any two orthogonal lines. So we let $\tau$ translate the atomic formula $d(a) \perp^{*} d(b)$ as $a \perp b$. Other atomic predications are handled analogously. Finally, we let $\tau$ commute with the connectives and quantifiers.

Is this 'reproduction' of meaning compatible with the laws of logic? Commenting on the translation of $d(a)=d(b)$ as $a \| b$, Frege raises a special instance of this question.

$[\mathrm{A}]$ re we not liable, through using such methods, to become involved in a conflict with the well-known laws of identity? (ibid.)

That is, what guarantee do we have that the contemplated 'reproduction' of meaning will be compatible with the logical laws of identity? As Frege observes, the concern can be addressed by observing, firstly, that the relation on which we abstract is an equivalence relation, and secondly, that this equivalence relation is a congruence with respect to any ordinary predicate $P$ that we are prepared to extend to a direction predicate $P^{*}$, in the way we extended the orthogonality predicate $\perp$ on lines to an orthogonality predicate $\perp^{*}$ on directions. ${ }^{31}$ What about logical laws other than those concerned with identity? Curiously, Frege does not discuss this. But it can be shown that these laws too are respected by the translation $\tau{ }^{32}$

As announced, I shall consider three different ways to develop the explanatory strategy associated with Frege's context principle. The crucial difference between these developments

\footnotetext{
${ }^{30} \mathrm{~A}$ formal definition is provided in (Linnebo, , ch. 6).

${ }^{31}$ Recall that an equivalence relation $\sim$ is a congruence with respect to a predicate $F$ just in case $F$ does not discriminate between $\sim$-equivalent objects; that is: $F\left(u_{1}, \ldots, u_{n}\right) \wedge u_{1} \sim v_{1} \wedge \ldots \wedge u_{n} \sim v_{n} \rightarrow F\left(v_{1}, \ldots, v_{n}\right)$.

${ }^{32}$ Again, see (Linnebo, , ch. 6).
} 
concerns their respective accounts of what the translation $\tau$ achieves.

\subsection{Holophrastic reductionism}

According to the first way to to develop the context principle, the point of the translation $\tau$ is to assign meanings to the partially interpreted sentences of the extended language $\mathcal{L}_{1}$. More precisely, each sentence $\phi$ of $\mathcal{L}_{1}$ is to mean just the same as the sentence $\tau(\phi)$ of the fully interpreted base language $\mathcal{L}_{0}$. Since each sentence of $\mathcal{L}_{1}$ is assigned a meaning expressed in the unproblematic base language $\mathcal{L}_{0}$, this is a reductionist view. And since each sentence is assigned a meaning only as a whole, not in virtue of any meanings assigned to its subsentential parts, the reductionism can be characterized as holophrastic.

Assume that a community uses $\mathcal{L}_{1}$ in accordance with the mentioned convention (as is surely possible). What have they achieved? They have in a systematic manner assigned a meaning to each sentence of the direction language. And as explained, we may assume that these meanings are inferentially related to one another so as to make all the 'right' inferences hold; that is, that the meanings assigned to the sentences of $\mathcal{L}_{1}$ are related to one another precisely as they would be if $\mathcal{L}_{1}$ was taken at face value, as concerned with directions. But do the direction terms of $\mathcal{L}_{1}$ really refer to directions? Dummett comments on a similar scenario as follows.

This may quite reasonably be taken as a sound method of justifying the use of names of directions: but it would naturally be construed, not as a demonstration that such names have reference in the same way as names of concrete objects, but as a way of explaining their use without ascribing reference to them. (Dummett, 1981a, pp. 499-500)

This assessment seems to me exactly right. True, the syntactic structure of $\mathcal{L}_{1}$ and the inferential relations between its sentences are precisely as if the language was about abstract directions. But in fact, the language is about no such things. For the meanings assigned to its sentences are concerned with lines only.

This result is important philosophically. It shows that it is possible to speak the same language as a platonist and correctly to make the same inferences in this language as the 
platonist without thereby having to be a platonist. ${ }^{33}$

I can think of only one way to resist the assessment just discussed, which is to adopt a deflationist conception of reference. Here is an example of such a conception. ${ }^{34}$

After all, what we state metalinguistically by "a" has reference' is just the object language, ' $(\exists x) x=a$ ' (Wright, 1983, p. 83)

This passage can scarcely be read as anything other than a commitment to a deflationist conception of reference on which it suffices for a singular term $t$ to refer that the object language sentence $\exists x(x=t)$ is true. If this was all it meant to ascribe reference to a term, then the holophrastic reductionist interpretation would indeed secure reference for all of its terms. However, this conception of reference is unlikely to have satisfied Frege and certainly should not satisfy us. Frege's question of how numbers are 'given to us' calls for a genuine relation between linguistic or mental representations and objects to which the representations refer, not just the truth of certain object language sentences. Indeed, by the time of the Grundgesetze, Frege himself had done groundbreaking work on the theory of reference, involving the discovery that reference is determined compositionally and his celebrated theory of sense and reference.

\subsection{Semantically constrained content recarving}

What goes wrong with the previous strategy is that the syntactic constituents of a sentence of the extended language are left without any direct semantic significance. In particular, singular terms and the identity predicate do not make their ordinary semantic contribution of standing for objects and the relation of identity respectively. Only whole sentences have been assigned a meaning.

In fact, there is reason to doubt that Frege had that strategy in mind. Discussing the idea that one side of an abstraction principle might be taken "to mean the same as" the other side,

\footnotetext{
${ }^{33}$ (Dummett, 1991a, pp. 191ff) aptly characterizes this sort of view as 'tolerant reductionism'. It is tolerant of the language and reasoning of the platonist yet gives this language a reductionist interpretation.

${ }^{34}$ To be clear, I do not claim that Wright invokes this conception in order to defend precisely the view that I have just described, only that it can be so invoked. Other examples include (Dummett, 1956, p. 41) and (Resnik, 1976, p. 43).
} 
he remarks that the relation of identity must be 'taken as already known' (Frege, 1953, § 65). ${ }^{35}$ It is reasonable to take this remark to express a requirement that is semantic in character, not merely inferential, namely that the identity predicate be given its ordinary semantic interpretation of standing for the relation of identity. For this semantic interpretation to work, the singular terms that flank the identity predicate must obviously be interpreted as referring to objects; otherwise, the identity relation would have no relata.

We may regard this revised strategy as a semantically constrained form of content recarving. Consider an abstraction principle. We want to assign the independently available meaning of the right-hand side to the left-hand side - but subject to a semantic constraint that was not present in the previous strategy, namely that the identity predicate be given its ordinary interpretation. The hope is that the assignment of meaning, subject to this added constraint, will suffice to determine the reference of the relevant abstraction terms. Metaphorically, the idea is to pour the meaning of each instance of the right-hand side into a mould provided by the logical form of the left-hand side, and in this way to fix the reference of the relevant singular terms. I believe this approach is roughly what Frege had in mind in Grundlagen $\S 64$ when he wrote about 'carving up' a content in different ways. ${ }^{36}$

Unfortunately, this revised strategy too faces serious problems. It suffices for present purposes to consider an exegetical problem. ${ }^{37}$ While the interpretation of the context principle as concerned with a semantically constrained form of content recarving arguably receives some support from the Grundlagen, it does not fit the Grundgesetze. ${ }^{38}$ This later work abandons the idea of content recarving in favor of transformations that need only preserve truth-value, while the context principle retains an important role. It follows that the mature Frege must have understood the context principle in a way that was not essentially tied to the idea of content recarving.

\footnotetext{
${ }^{35}$ The same view is held in the Grundgesetze; see (Frege, 2013, I, $\S \S 3$ and 9).

${ }^{36}$ Strictly speaking, Frege writes about 'splitting up' (zerspalten) the content of a relation symbol. (Thanks to Philip Ebert and Bob Hale for discussion.) However, this 'splitting up' must affect the content of the entire sentence; otherwise it would not make any sense to talk about 'dividing' the content of the relation symbol between the two objects on which we abstract.

${ }^{37}$ In fact, even when exegesis is set aside, the present strategy for explaining the reference of abstraction terms is problems, or so I argue in (Linnebo, , ch. 7).

${ }^{38}$ See (Ebert, 2016) for some interesting concerns about Frege's commitment to the idea of content recarving even in the Grundlagen.
} 


\subsection{Towards a metasemantic interpretation}

The previous two explanatory strategies take their inspiration from the Grundlagen. Let us now consider how Frege understood the context principle in the Grundgesetze.

I wish to begin with a problem that lies right at the heart of the context principle as I understand it. Consider an abstraction term, say ' $d(l)$ ', where ' $l$ ' is the name of some particular line. Suppose that doubt arises as to whether the abstraction term refers. How can this doubt be assuaged? One option is to look to Frege's semantic theory, which tells us that ' $d(l)$ ' refers to the direction of the line to which ' $l$ ' refers. But this specification of a referent involves an analogous abstraction term in the metalanguage, which is just as vulnerable to the doubt in question. Frege's context principle is meant to provide a more independent - and therefore more satisfying - way to assuage the doubt. According to the generalized context principle, it suffices for an expression to have reference that all appropriate contexts in which the expression occurs also refer. In order to verify that ' $d(l)$ ' satisfies this sufficient condition, we invoke our translation $\tau$, which renders each sentence involving direction terms as a materially equivalent sentence involving no such terms. Since all the sentences in the range of $\tau$ have reference, so do the sentences in its domain. This means that our abstraction term ' $d(l)$ ' satisfies the sufficient condition for having reference.

There is something deeply puzzling about this way to defend the referentiality of the term. The sufficient condition for the term ' $d(l)$ ' to refer does not mention any referent of the term or appear to involve one in any other way. By design, the condition is concerned only with the referentiality of complete sentences not involving the term. How, then, can the condition suffice for the existence of a referent? Imagine that someone proposes a sufficient condition for being married that does not mention or in any other way involve a spouse. We would of course be inclined to reject the condition. Why should the proposed sufficient condition for having reference be any better? This is, I think, the hardest and most fundamental of all the problems that confront the context principle. As far as I can see, no solution is worked out in any of Frege's writings.

I therefore ask the reader's indulgence to end with a somewhat speculative suggestion about the kind of solution that Frege might have intended. The context principle is concerned 
with a metasemantic question, namely how reference is constituted. It is not a primitive metaphysical fact that an expression refers to some object. There are some more basic facts in virtue of which the relation of reference obtains. Perhaps the more basic facts in virtue of which an abstraction term refers to an abstract object need not mention or otherwise involve this abstract object. Some other examples suggest that this is at least a possibility. Consider the relation that obtains between me and a bank account that I own. It is not a primitive metaphysical fact that this relation obtains. Instead, the fact obtains in virtue of some complex network of psychological and social facts. There is no reason why these more basic facts in virtue of which I own the account should themselves mention or in any obvious way involve the account. What is constituted by these more basic facts is not only a particular relation of ownership but also one of the relata.

Of course, there are important differences between this case and that of reference to mathematical objects. In particular, where a bank account depends on human thought and actions for its existence, mathematical objects do not. So if this strategy is to work, mathematical objects would have to be constituted, as it were, along with their modal profile as necessary existents. If such an account can be developed, it would provide just what the Grundgesetze needs. There would be genuine reference to abstract objects, just as there is genuine ownership of bank accounts. Simultaneously, the sufficient condition for a term to refer would not itself mention any referent. This would of course give the account a reductionist flavor. But unlike the holophrastic reductionism criticized in Section 6.2, the alternative form of reductionism currently envisaged would not deny that there is reference to abstract objects. This alternative would locate the reductionism, not at the syntactic or semantic level, but at the metasemantic level, as the claim that reference to abstract objects is constituted by more basic facts that do not themselves involve the relevant abstract objects. Admittedly, this proposal is highly programmatic. The purpose of this book is to develop-without any regard for exegetical issues - a view of the sort that the proposal requires.

Any version of metasemantic reductionism will no doubt strike some readers as a less robust form of realism than what they associate with Frege. So is my proposed interpretation compatible with what Frege writes? I believe it is. The discussion following Frege's criticism of 
creative definitions is once again relevant. Frege returns in $\S 147$ to the question of whether his procedure for 'converting' an instance of the left-hand side of Basic Law V to the corresponding instance of the right-hand side should be seen as a form of creation.

Can our procedure be called a creation? The discussion of this question can easily degenerate into a quarrel about words. In any case, our creation, if one wishes so to call it, is not unconstrained and arbitrary, but rather the way of proceeding, and its permissibility, is settled once and for all. And with this, all the difficulties and concerns that otherwise put into question the logical possibility of creation vanish; and by means of our value-ranges we may hope to achieve everything that these other approaches fall short of. (Frege, 2013, II, § 147)

This passage is remarkably concessive. One would have expected Frege to come out swinging against anything that smacks of creative definition. Instead, he concedes that his own procedure might, if one so wishes, be called a creation and rather holds up as the key advantage of his account over those of his creationist opponents that it alone is systematic and rigorous. This suggests that there may not, after all, be a clash between Frege's criticism of creative definitions and the interpretation of the context principle that I have proposed. What matters is that the procedure not be 'unconstrained and arbitrary'. And these requirements are satisfied on the view developed throughout this book, or so I argue.

\section{Conclusion}

While it is widely agreed that the context principle plays a central role in the logicist project of the Grundlagen, there are arguments that suggest there is no room for the principle in the Grundgesetze. I have rebutted these arguments and shown that the principle survives into the Grundgesetze, where it retains a central role in what is essentially the same explanatory project. Of course, there are changes to both the context principle and the argument in which it figures. But these changes are brought about by other developments in Frege's thinking, especially about semantics, and do not bespeak any dissatisfaction with the context principle per se. 
The longevity of the context principle in Frege's philosophy is of obvious scholarly interest and provides invaluable help in making sense of this intriguing but elusive principle. One lesson that has emerged from our discussion is that the context principle must be separated from the problematic idea of content recarving. This is surprising in light of the close connection between the two ideas in the Grundlagen and in so much of the subsequent literature.

Another lesson is that the context principle must be separated from the deflationist conception of reference, which, as I have argued, sits poorly with the general thrust of Frege's mature thinking about semantics. Nevertheless, the context principle in the Grundgesetze has an internalist character - in the sense that the reference of mathematical singular terms (and perhaps others too) cannot ultimately be explained by 'giving' the referent of the term in a more fundamental way. The explanation will instead have to turn on considerations in which the intended referent does not play any direct role. The availability of such reductive explanations suggests a respect in which the objects that serve as referents are metaphysically 'lightweight'. 39,40

\section{References}

Boolos, G. (1990). The standard of equality of numbers. In Boolos, G., editor, Meaning and Method: Essays in Honor of Hilary Putnam, pages 261-278. Harvard University Press, Cambridge, MA. Reprinted in (Boolos, 1998).

Boolos, G. (1998). Logic, Logic, and Logic. Harvard University Press, Cambridge, MA.

Burge, T. (2005). Truth, Thought, Reason: Essays on Frege. Oxford University Press, Oxford.

Dummett, M. (1956). Nominalism. Philosophical Review, LXV:491-505. repr. in (Dummett, 1978).

Dummett, M. (1978). Truth and Other Enigmas. Harvard University Press, Cambridge, MA.

Dummett, M. (1981a). Frege: Philosophy of Language. Harvard University Press, Cambridge, MA, second edition

Dummett, M. (1981b). The Interpretation of Frege's Philosophy. Harvard University Press, Cambridge, MA. Dummett, M. (1991a). Frege: Philosophy of Mathematics. Harvard University Press, Cambridge, MA.

\footnotetext{
${ }^{39}$ This suggestion is developed in (Linnebo, ).

${ }^{40}$ Work on this paper was started in 2007 during a period of AHRC-sponsored research leave and has continued in irregular bursts throughout the following decade. I am grateful for comments from Christian Beyer, Patricia Blanchette, Philip Ebert, Anthony Everett, Bob Hale, Richard Heck, Keith Hossack, Hannes Leitgeb, Marcus Rossberg, Ian Rumfitt, Tolgahan Toy, Crispin Wright, anonymous referee, and audiences in Aberdeen, Besse-en-Chandesse, London, Milano, Munich, Oslo, Philadelphia (Eastern APA), and Stirling.
} 
Dummett, M. (1991b). The Logical Basis of Metaphysics. Harvard University Press, Cambridge, MA.

Dummett, M. (1995). The context principle: Centre of frege's philosophy. In Max, I. and Stelzner, W., editors, Logik und Mathematik: Frege-Kolloquium Jena 1993. de Gruyter, Berlin.

Dummett, M. (1998). Neo-fregeans in bad company? In Schirn, M., editor, Philosophy of Mathematics Today. Clarendon, Oxford.

Ebert, P. (2015). Dummett's criticism of the context principle. Grazer Philosophische Studien, 92:23-50.

Ebert, P. A. (2016). Frege on sense identity, basic law V, and analysis. Philosophia Mathematica, 24(1):9-29.

Frege, G. (1953). Foundations of Arithmetic. Blackwell, Oxford. Transl. by J.L. Austin.

Frege, G. (1956). The thought: A logical inquiry. Mind, 65(259):289-311.

Frege, G. (1963). Compound thoughts. Mind, 72:1-17. Originally published in 1923.

Frege, G. (1979). Posthumous Writings. Blackwell, Oxford.

Frege, G. (2013). Basic Laws of Arithmetic. Oxford University Press, Oxford. Translated by Philip A. Ebert and Marcus Rossberg.

Hale, B. (1997). Grundlagen §64. Proceedings of the Aristotelian Society, 97(3):243-61. Reprinted with a postscript in (Hale and Wright, 2001).

Hale, B. (2007). Kit Fine on The Limits of Abstraction. Travaux de Logique, 18:103-129.

Hale, B. and Wright, C. (2000). Implicit definition and the a priori. In Boghossian, P. and Peacocke, C., editors, New Essays on the A Priori. Oxford University Press, Oxford. Reprinted in (Hale and Wright, 2001)

Hale, B. and Wright, C. (2001). Reason's Proper Study. Clarendon, Oxford.

Heck, Jr., R. G. (1997). Grundgesetze der Arithmetik I §§29-32. Notre Dame Journal of Formal Logic, 38(3):437-474.

Heck, Jr., R. G. (2012). Reading Frege's Grundgesetze. Oxford University Press.

Janssen, T. M. V. (2001). Frege, contextuality and compositionality. Journal of Logic, Language and Information, 10(1):115-136.

Kripke, S. A. (1975). Outline of a theory of truth. Journal of Philosophy, 72(19):690-716.

Künne, W. (2010). Die Philosophische Logik Freges. Klostermann, Frankfurt am Main.

Linnebo, Ø. Thin Objects: An Abstractionist Account. Oxford University Press, Oxford. Forthcoming.

Linnebo, Ø. (2004). Frege's proof of referentiality. Notre Dame Journal of Formal Logic, 45(2):73-98.

Parsons, C. (1965). Frege's theory of number. In Black, M., editor, Philosophy in America, pages 180-203. Cornell University Press, Ithaca, NY. Reprinted in (Parsons, 1983).

Parsons, C. (1983). Mathematics in Philosophy. Cornell University Press, Ithaca, NY. 
Pelletier, F. J. Did Frege believe Frege's principle?

Quine, W. (1953). From a Logical Point of View. Harvard University Press, Cambridge, MA.

Resnik, M. (1967). The Context Principle in Frege's Philosophy. Philosophy and Phenomenological Research, 27:356-365.

Resnik, M. (1976). Frege's context principle revisited. In Schirn, M., editor, Studien zu Frege III: Logik und Semantik, pages 35-49. Frommann-Holzboog, Stuttgart.

Rumfitt, I. (2011). Truth and the determination of content: Variations on themes from Frege's Logische Untersuchungen. Grazer Philosophische Studien, 82(1):3-48.

Wright, C. (1983). Frege's Conception of Numbers as Objects. Aberdeen University Press, Aberdeen.

Wright, C. (1998a). The harmless impredicativity of $N^{=}$(Hume's Principle). In Schirn, M., editor, Philosophy of Mathematics Today. Clarendon, Oxford.

Wright, C. (1998b). Response to Michael Dummett. In Schirn, M., editor, Philosophy of Mathematics Today. Clarendon, Oxford. 